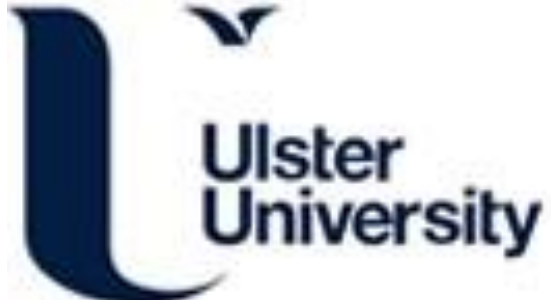

Towards conversational technology to promote, monitor and protect mental health

Callejas, Z., Griol, D., Benghazi, K., Noguera, M., Torres, M. I., Justo, R., Esposito, A., Cordasco, G., Bond, RR., Mulvenna, M., Ennis, E., O'Neill, S., Zheng, H., Kraus, M., Wagner, N., Minker, W., McConvey, G., Hemmje, M., Fuchs, M., ... Chollet, G. (2021). Towards conversational technology to promote, monitor and protect mental health. 148-150. Paper presented at IberSpeech, Valladolid, Spain. https://doi.org/10.21437/lberSPEECH.202132

Link to publication record in Ulster University Research Portal

Publication Status:

Published (in print/issue): 24/03/2021

DOI:

https://doi.org/10.21437/lberSPEECH.2021-32

Document Version

Author Accepted version

\section{General rights}

Copyright for the publications made accessible via Ulster University's Research Portal is retained by the author(s) and / or other copyright owners and it is a condition of accessing these publications that users recognise and abide by the legal requirements associated with these rights.

\section{Take down policy}

The Research Portal is Ulster University's institutional repository that provides access to Ulster's research outputs. Every effort has been made to ensure that content in the Research Portal does not infringe any person's rights, or applicable UK laws. If you discover content in the Research Portal that you believe breaches copyright or violates any law, please contact pure-support@ulster.ac.uk. 


\title{
Towards conversational technology to promote, monitor and protect mental health
}

\author{
Zoraida Callejas $^{1}$, David Griol ${ }^{1}$, Kawtar Benghazi ${ }^{1}$, Manuel Noguera ${ }^{1}$, María Inés Torres ${ }^{2}$, Raquel \\ Justo $^{2}$, Anna Esposito ${ }^{3}$, Gennaro Cordasco ${ }^{3}$, Raymond Bond ${ }^{4}$, Maurice Mulvenna ${ }^{4}$, Edel Ennis ${ }^{4}$, \\ Siobhan O'Neill ${ }^{4}$, Huiru Zheng ${ }^{4}$, Matthias Kraus ${ }^{5}$, Nicolas Wagner ${ }^{5}$, Wolfgang Minker ${ }^{5}$, Gavin \\ McConvey ${ }^{6}$, Matthias Hemmje ${ }^{7}$, Michael Fuchs ${ }^{7}$, Neil Glackin ${ }^{8}$, Gérard Chollet ${ }^{8}$
}

${ }^{1}$ University of Granada, Granada, Spain,

${ }^{2}$ University of the Basque Country, Bilbao, Spain

${ }^{3}$ Università degli Studi della Campania "Luigi Vanvitelli”, Casserta, Italy

${ }^{4}$ Ulster University, Belfast, Northern Ireland

${ }^{5}$ Ulm University, Ulm, Germany

${ }^{6}$ Action Mental Health, Newtonards, Northern Ireland

${ }^{7}$ GLOBIT, Pfungstadt, Germany

${ }^{8}$ Intelligent Voice, London, United Kingdom

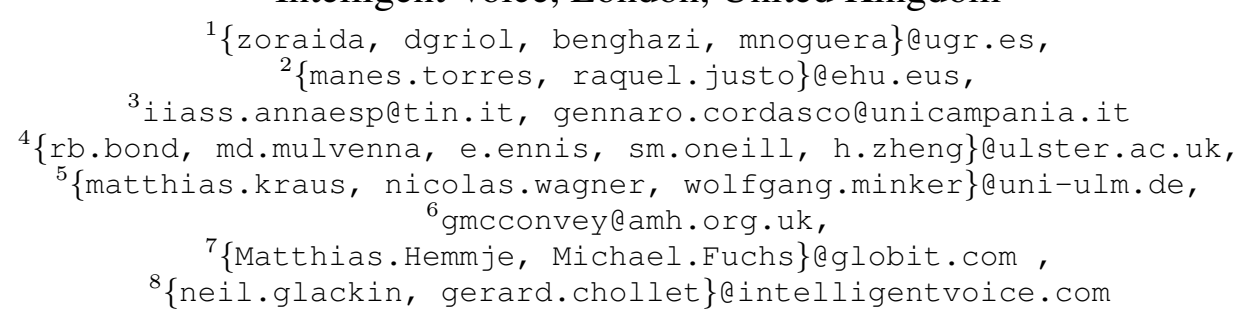

\begin{abstract}
This paper presents a general overview of the H2020MSCA-RISE project MENHIR (Mental health monitoring through interactive conversations), which aim is to explore the possibilities of conversational technologies (chatbots) to understand, promote and protect mental health and assist people with anxiety and mild depression manage their conditions. MENHIR started on February 2019 and will have a duration of 4 years. Its consortium brings together 8 partners including universities, a non-profit organization and companies.

Index Terms: mental e-health, conversational systems, chatbots, dialogue systems, wellbeing
\end{abstract}

\section{Introduction}

Mental wellbeing is a very important aspect of health. New technology solutions such as chatbots are potential channels for supporting and coaching users to maintain a healthy lifestyle $[1,2]$ and in particular a good state of mental wellbeing. Conversational systems ${ }^{1}$ have the added value of providing social conversations and coaching 24/7 outside from conventional mental health services.

The MENHIR project (http://menhir-project. $\mathrm{eu}$ ) has received funding from the European Union's Horizon 2020 research and innovation programme under grant agreement No 823907, concretely in the funding scheme Marie

\footnotetext{
${ }^{1}$ We will refer to them as chatbots. Although chatbots are frequently used to describe conversational systems with which to maintain open chit-chat (non task-oriented) conversations, we use the term as a synonym of a general conversational system or dialogue system. We have adopted this term because it is more recognizable by the end users who are co-creating the technology in MENHIR.
}

Sklodowska-Curie Research and Innovation Staff Exchange (MSCA-RISE) 2018.

MENHIR aims at engaging people in the management of self-care and providing a more flexible, immediate and sustained complement to under pressure mental health services [3]. The MENHIR chatbot technology provides symptom and mood management, personalized support and motivation, coping strategies, mental health education, signposting to online resources and complementing the support received by local services.

The technology developed will be able to process emotional and mood patterns from different aspects of the voice recorded (e.g. from the tone of voice or speed of speech). This information facilitates rapid intervention and appropriate feedback such as prompts and supportive messages. It will also generate personalized conversations with the users to motivate and engage them at any time and especially in between visits to their local services.

The limitations of previous technology are the high dropout rates due in part to a lack of interactive features and appropriate prompts, difficulties for people with low levels of technological literacy and failure to provide human support. The MENHIR chatbot technology aims to address these difficulties being more usable and intuitive since it simulates everyday human to human conversation.

The project will also generate data on how people with anxiety and depression interact with and respond to this technology, and about the benefits it brings to them, which will be useful to understand the role that chatbots can play to promote mental health. 


\section{Consortium}

The MENHIR consortium is comprised of eight partners:

- University of Granada, Spain (coordinator, academic).

- University of the Basque Country, Spain (academic).

- Università degli Studi della Campania "Luigi Vanvitelli", Italy (academic).

- Ulster University, United Kingdom (academic).

- Ulm University, Germany (academic).

- Action Mental Health, United Kingdom (non-profit).

- GLOBIT - Globale Informationstechnik GmbH, Germany (company).

- Intelligent Voice Limited, United Kingdom (company).

\section{Objectives}

MENHIR has been funded by the RISE programme, which aim is the sharing of knowledge and ideas across countries, sectors and disciplines. Thus, one of the main objectives of the project is to develop new capabilities and skills for MENHIR participants.

The staff participating in MENHIR will create new opportunities for IT researchers to increase their impact rooting technology in a deeper understanding of their prospective users, while psychologists and mental health experts will gain knowledge of the technological tools that can help them improve their clients' lives.

The main scientific objectives are to: i) establish the strengths, limitations and requirements of mental health chatbots; ii) develop the MENHIR chatbot technology collaborating with people who suffer anxiety and/or mild depression; and iii) understand how users engage with chatbot technology and how it may affect their well-being over time.

\section{Progress beyond the state of the art and potential impacts}

MENHIR will progress beyond the state of the art by:

- Determining the most practical scales for monitoring mental wellbeing.

- Compiling mental health coping strategies, prompts, relevant digital content and resources.

- Providing new evidence on how to perform a crossmodal analysis of the interactional exchanges to automatically recognize the users' emotional state and anxiety level.

- Defining how to track mood and anxiety from usersystem interactions and how to represent user progress in a computational model.

- Providing novel approaches to generate computerized models of the users to adapt decision making, conversational models and communication styles.

- Finding novel approaches to make chatbot interaction personalized to their users, taking into consideration that they are heterogeneous in terms of age, gender, mental health condition, progression and responses to system's strategies.

- Identifying adequate dialogue management strategies based on the user progress and the history of the usersystem interaction.
- Establishing adequate proactivity approaches and disparate conversation structures to manage conversation and favour user self-disclosure.

- Establishing new methods to favour user-system trust through active listening.

- Generating good practices for data storage, annotation and sharing.

- Generating good practices for participatory research based on co-creation.

These results are being documented in several reports that are being delivered during the project, corresponding to: the chatbot strategy for mental health monitoring, the characterization and analysis of human-to-human interaction, the crossmodal analysis and annotations of mood and anxiety levels, user modelling (including mood and anxiety level), and conversation modelling.

MENHIR will have a considerable impact, as it presents a unique research and innovation plan which tackles several challenges from different areas merging multiple expertise of academic and non-academic members with a background in Psychology, Cognitive Sciences, Computer Science and Engineering. MENHIR will help to achieve the vision that should be accomplished by 2025 according to the Strategic Roadmap for the area of multimodal conversational interaction technologies [4], as it will help to achieve several of the multiple goals defined in the roadmap, including:

- Multimodal conversational interfaces with the potential to adapt automatically to the user and to the user's state.

- Make predictions and recommendations based on personal data with a sensitive management of information.

- Domain-specific or context-based personal assistants.

- Dialogue modelling and management with cognitive human modelling, flexible domain adaptation and socially acceptable dialogues.

- Ability to understand emotions, sentiment and social signals as well as the meaning of the spoken word.

\section{Recent advances and current work}

MENHIR chatbot technology is a complement to the efforts of mental health service providers, so it was important that all MENHIR members became aware of the ways in which technology can be useful to them and their clients and avoid introducing functionality that could be unintentionally detrimental for the user's wellbeing. This was achieved during mutual visits (secondments)

In MENHIR we have adopted a co-creation methodology. This means that the prospective users participate in the design and development of technology not as passive recipients, but as active members that are engaged from the beginning in the relevant decisions. Prospective users are represented in MENHIR by clients of Action Mental Health (AMH) across its different services in Northern Ireland. A co-creation workshop was performed on June 2019. The participants were 9 AMH clients, a key worker and 3 MENHIR researchers who presented the project and acted as facilitators. This was a lively and very interesting conversation that provided relevant insight for the project.

Among the results of the workshop, we elaborated a list of the perceived strengths and limitations of the technology and 
also a study of the main topics and themes covered, which included the challenges faced by people such as isolation and difficulty for honest disclosure, symptom recognition, continuous monitoring, disclosure facilitation, companionship, risk detection, personalization, configurable proactiveness, user access to information, privacy, vulnerability, cost and access to the technology and use of the chatbot. The results are the co-created functional requirements and a number of use case scenarios that can be of interest to guide future development of chatbots in the mental health domain are described in further detail in [5].

The results of the co-creation together with a detailed analysis of the scientific literature about mental health monitoring systems and multimodal emotion and mood recognition, allowed to define the scenarios in which it will be used, which will be mainly four: intelligent reminder, diary, progress evaluation and risk detection.

In the intelligent reminder scenario, the chatbot will remind users to engage in the activities planned for them. The reminder will be capable of making adequate decisions on how, when and if at all to remind and motivate users to stick to their plans without being obtrusive. This will be achieved by means of a rich user model based on the user's objectives and engagement, challenges that are pervasive to different types of multimodal ecoaches [6]. Adaptive, trustworthy dialogue management will be key to this task $[7,8]$, for which different models will be put in place an evolved as more data is available $[9,10]$. In relation to this, we are conducting several studies related to the acceptance of different aspects of the interaction with a conversational system in multiple settings. [11, 12].

The diary use case provides users the possibility to talk to the system about their day. The chatbot provides follow-up questions to build rapport and keep the user engaged and talking. The goal is to offer companionship and record the user interventions. Then, we will develop technology that will be able to infer the user's emotional state from the recordings using paralinguistic information considering the different input sources and interaction settings [13].

In MENHIR we are devoting a great effort to data collection, annotation, representation and sharing, see e.g. [14]. We have performed and annotated anonymized recordings of $\mathrm{AMH}$ clients and a control group that we are currently processing to obtain relevant features for user state recognition.

The progress evaluation use case will be based on the adaptation of questionnaires designed to assess mental health. This serves to address the need for continuous monitoring using standardized and widely recognized questionnaires, and will be used to check whether the state recognized automatically from the recorded entries to the user diary is reliable.

Risk detection functionalities are pervasive to the previous scenarios and consist in detecting and notifying risks based on predefined words and phrases and raise an alert.

\section{Acknowledgements}

This research has received funding from the European Union's Horizon 2020 research and innovation programme under grant agreement No 823907 (MENHIR: Mental health monitoring through interactive conversations https:// menhir-project.eu).

\section{References}

[1] A. Benítez-Guijarro, Z. Callejas, M. Noguera, and K. Benghazi, "Architecting dietary intake monitoring as a service combining
NLP and IoT," Journal of Ambient Intelligence and Humanized Computing, 2019.

[2] D. Griol and Z. Callejas, "Mobile Conversational Agents for Stroke Rehabilitation Therapy," in 2019 IEEE 32nd International Symposium on Computer-Based Medical Systems (CBMS), 2019, pp. 513-518.

[3] Z. Callejas, K. Benghazi, M. Noguera, M. I. Torres, and R. Justo, "MENHIR: Mental health monitoring through interactive conversations," Procesamiento del Lenguaje Natural, vol. 63, 2019.

[4] S. Renals, J. Carletta, K. Edwards, H. Bourlard, P. Garner, A. Popescu-Belis, D. Klakow, A. Girenko, V. Petukova, P. Wacker, A. Joscelyne, C. Kompis, S. Aliwell, W. Stevens, and Y. Sabbah, "ROCKIT: Roadmap for Conversational Interaction Technologies," in Proceedings of the 2014 Workshop on Roadmapping the Future of Multimodal Interaction Research including Business Opportunities and Challenges, ser. RFMIR '14. New York, NY, USA: Association for Computing Machinery, 2014, pp. 39-42.

[5] A. Benítez-Guijarro, R. Bond, F. Booth, Z. Callejas, E. Ennis, A. Esposito, M. Kraus, G. McConvey, M. McTear, M. Mulvenna, C. Potts, L. Pragst, R. Turkington, N. Wagner, and H. Zheng, "Co-creating Requirements and Assessing End-User Acceptability of a Voice-Based Chatbot to Support Mental Health: A Thematic Analysis of a Living Lab Workshop," in Conversational Dialogue Systems for the Next Decade, L. F. D'Haro, Z. Callejas, and S. Nakamura, Eds. Singapore: Springer, 2021, pp. 201-212.

[6] L. Angelini, M. El Kamali, E. Mugellini, O. Abou Khaled, Y. Dimitrov, V. Veleva, Z. Gospodinova, N. Miteva, R. Wheeler, Z. Callejas, D. Griol, K. Benghazi, M. Noguera, P. Bamidis, E. Konstantinidis, D. Petsani, A. Beristain Iraola, D. I. Fotiadis, G. Chollet, I. Torres, A. Esposito, and H. Schlieter, "First Workshop on Multimodal e-Coaches," in Proceedings of the 2020 International Conference on Multimodal Interaction, ser. ICMI '20. New York, NY, USA: Association for Computing Machinery, 2020, pp. 890-892.

[7] D. Griol, Z. Callejas, J. M. Molina, and A. Sanchis, "Adaptive dialogue management using intent clustering and fuzzy rules," Expert Systems, vol. 38, no. 1, 2021.

[8] M. Kraus, P. Seldschopf, and W. Minker, "Towards the Development of a Trustworthy Chatbot for Mental Health Applications," in 27th International Conference on Multimedia Modeling. Prague, Czech Republic: Springer, 2021.

[9] D. Griol and Z. Callejas, "Discovering Dialog Rules by Means of an Evolutionary Approach,” in Interspeech 2019. ISCA, 2019, pp. 1473-1477.

[10] A. Esposito, M. Faundez-Zanuy, F. C. Morabito, and E. Pasero, "Some Note on Artificial Intelligence," in Neural Approaches to Dynamics of Signal Exchanges, ser. Smart Innovation, Systems and Technologies. Singapore: Springer, 2020, pp. 3-8.

[11] A. Esposito, T. Amorese, M. Cuciniello, A. M. Esposito, A. Troncone, M. I. Torres, S. Schlögl, and G. Cordasco, "Seniors' Acceptance of Virtual Humanoid Agents," in Ambient Assisted Living. Springer, Cham, 2018, pp. 429-443.

[12] A. Esposito, T. Amorese, M. Cuciniello, M. T. Riviello, A. M. Esposito, A. Troncone, and G. Cordasco, "The Dependability of Voice on Elders' Acceptance of Humanoid Agents," in Interspeech 2019. ISCA, 2019, pp. 31-35.

[13] A. Benítez-Guijarro, Z. Callejas, M. Noguera, and K. Benghazi, "Coordination of Speech Recognition Devices in Intelligent Environments with Multiple Responsive Devices," Proceedings, vol. 31 , no. 1 , p. $54,2019$.

[14] B. Vu, M. deVelasco, P. Mc Kevitt, R. Bond, R. Turkington, F. Booth, M. Mulvenna, M. Fuchs, and M. Hemmje, "A Content and Knowledge Management System Supporting Emotion Detection from Speech," in Conversational Dialogue Systems for the Next Decade, ser. Lecture Notes in Electrical Engineering, L. F. D'Haro, Z. Callejas, and S. Nakamura, Eds. Singapore: Springer, 2021, pp. 369-378. 